\title{
Antibiotic use and the risk of rheumatoid arthritis: a population-based case-control study
}

\author{
Alyshah Abdul Sultan', Christian Mallen', Sara Muller ${ }^{1}$, Samantha Hider ${ }^{1,2}$, Ian Scott ${ }^{1,2}$, Toby Helliwell ${ }^{1}$ and \\ Lindsay J. Hall ${ }^{3^{*}}$ (D)
}

\begin{abstract}
Background: Antibiotic-induced disturbances of the human microbiota have been implicated in the development of chronic autoimmune conditions. This study aimed to assess whether antibiotic use is associated with the onset of rheumatoid arthritis (RA).

Methods: A nested case-control study was conducted utilising data from the primary care Clinical Practice Research Datalink (CPRD). Patients with an incident diagnosis of RA were identified (1995-2017). Each case was matched on age, gender, and general practice to $\geq 5$ controls without RA. Conditional logistic regression was used to examine previous antibiotic prescriptions and RA onset after controlling for confounding factors.

Results: We identified 22,677 cases of RA, matched to 90,013 controls, with a median follow-up of 10 years before RA diagnosis. The odds of developing RA were $60 \%$ higher in those exposed to antibiotics than in those not exposed (OR 1.60; 95\% Cl 1.51-1.68). A dose- or frequency-dependent association was observed between the number of previous antibiotic prescriptions and RA. All classes of antibiotics were associated with higher odds of RA, with bactericidal antibiotics carrying higher risk than bacteriostatic (45\% vs. 31\%). Those with antibiotic-treated upper respiratory tract (URT) infections were more likely to be RA cases. However, this was not observed for URT infections not treated with antibiotics. Antifungal $(\mathrm{OR}=1.27$; $95 \% \mathrm{Cl} 1.20-1.35)$ and antiviral $(\mathrm{OR}=1.19 ; 95 \% \mathrm{Cl} 1.14-$ 1.24) prescriptions were also associated with increased odds of RA.
\end{abstract}

Conclusion: Antibiotic prescriptions are associated with a higher risk of RA. This may be due to microbiota disturbances or underlying infections driving risk. Further research is needed to explore these mechanisms.

\section{Background}

Antibiotics are prescribed to treat a wide range of bacterial infections including respiratory, gastrointestinal (GI), and urinary tract infections. In the UK, around $30 \%$ of all patients registered in primary care receive at least one antibiotic prescription per year [1]. As well as targeting bacterial pathogens, antibiotics can also disturb the gut microbiota. This highly diverse and dynamic microbial ecosystem plays an integral part in human health, modulating host metabolism and immunity [2-4]. Importantly, the microbiota can be influenced by numerous factors, with antibiotic treatment suggested as among the most

\footnotetext{
* Correspondence: lindsay.hall@quadram.ac.uk; Lindsay.Hall@quadram.ac.uk ${ }^{3}$ Gut Microbes \& Health Programme, Quadram Institute Bioscience, Norwich Research Park, Norwich NR4 7UQ, UK

Full list of author information is available at the end of the article
}

significant [5]. Antibiotic treatment can significantly disturb the gut, oral, and skin microbiota, leading to an immediate reduction in microbial abundance and species diversity. There is a significant body of data indicating that antibiotic usage, particularly during childhood, is a major risk factor for increasing susceptibility to infections and development of atopy and inflammatory bowel diseases (ulcerative colitis, Crohn's disease) [6]. More recently, studies indicate antibiotic usage appears to increase the risk of autoimmune conditions, including type 1 diabetes, autoimmune liver disease, and juvenile idiopathic arthritis (JIA) [7-10].

RA is a chronic autoimmune inflammatory disease, which is characterised by autoimmune antibody production, which directly leads to bone joint destruction, and associated RA pathology. One of the autoantibodies associated with RA (antibodies to citrullinated peptide

(c) The Author(s). 2019 Open Access This article is distributed under the terms of the Creative Commons Attribution 4.0 International License (http://creativecommons.org/licenses/by/4.0/), which permits unrestricted use, distribution, and reproduction in any medium, provided you give appropriate credit to the original author(s) and the source, provide a link to the Creative Commons license, and indicate if changes were made. The Creative Commons Public Domain Dedication waiver (http://creativecommons.org/publicdomain/zero/1.0/) applies to the data made available in this article, unless otherwise stated. 
antigens (ACPA) ) is produced in response to bacterial components that closely mimic host cell receptors, e.g. Porphyromonas gingivalis and Aggregatibacter actinomycetemcomitans $[11,12]$. Indeed, prior infections, and gingivitis in particular, have been identified as potential risk factors for RA development [13]. Notably, gingivitis is a very common oral infectious disease in the UK population, with most adults having some form of gum disease [14]. However, the proportion of this population who develop RA is extremely low, suggesting other aetiological factors are at play. Several experimental studies have linked the microbiota to the development of inflammatory arthritis. Germ-free mouse models do not develop inflammatory arthritis, and certain bacteria (e.g. segmented filamentous bacteria, a mouse-specific taxa) have been shown to induce inflammatory $\mathrm{T}_{\mathrm{H}} 17$ responses, which may drive associated immune-mediated pathology and joint destruction $[15,16]$. In humans, previous studies have indicated that there are differences in microbiota composition between healthy and disease cohorts. Although RA microbiota profiles reported have varied between different countries [17, 18], all studies have indicated reduced microbial diversity, which may be associated with external factors such as antibiotic usage. Thus, the aim of this study was to investigate the association between antibiotic prescriptions and the onset of RA using a large, UK-based, primary care dataset.

\section{Methods}

\section{Data source and study population}

We used the Clinical Practice Research Datalink (CPRD), a large database containing UK primary care medical records of anonymised patients. This includes coded symptoms, diagnoses, test results, referrals, and prescriptions. CPRD covers more than $7 \%$ of the UK population and is a representative of the general UK population in terms of age and sex [19]. Practices contributing data to the CPRD receive training on recording information, and the database is subjected to quality checks. Data are only used when a practice has reached a certain standard of quality. This is defined as being up to standard (UTS). Prescriptions, test results, and referrals are recorded automatically when requested by the treating clinician. It can be expected that all reasons for consultation of any significance are also recorded.

We identified individuals with a first-ever recorded diagnosis of RA between 1990 and 2017. Diagnosis of RA was ascertained by Read Codes, which have been previously shown to be accurate with a high positive predictive value $[20,21]$. Each RA case was assigned an index date corresponding to the incident diagnosis date and individually matched to up to five controls on the year of birth, gender, and general practice. Those with less than 5 years of follow-up before the index date were excluded.

\section{Antibiotic prescriptions}

In UK primary care, all prescriptions are recorded automatically when issued by the clinician, including a code for the chapter of the British National Formulary (BNF) under which they were issued. Therefore, all primary care prescriptions appear in the CPRD. All antibiotic prescriptions recorded more than 1 year before the index date were identified using relevant British National Formulary (BNF) chapter headings. We only focused on systemic oral antibiotics, excluding topical antibiotics. Individuals were considered exposed if they received one or more antibiotic prescriptions more than a year before the index date. Antibiotics prescribed in the year before the RA diagnosis were not considered, as they may be prescribed for early RA symptoms.

\section{Covariates}

For cases and controls, we extracted information on body mass index (BMI), alcohol consumption, and smoking status (current or ex-smoker/never smoker) using the latest measure before the index date. BMI was categorised into four categories: underweight (BMI < 18.5), normal (18.5$24.9)$, overweight (25-29.9), and obese $(\geq 30)$. Charlson index was used to quantify comorbidities before the index date. Finally, we extracted information on commonly occurring infections (upper and lower respiratory tract, urinary tract, GI) before the index date.

Code lists used to define exposures, outcome, and covariates are available from keele.ac.uk/mrr.

\section{Statistics analysis}

Characteristics of the cases and controls were summarised using descriptive statistics (frequencies and percentages). We used conditional logistic regression to assess the association between antibiotic exposure and the risk of incident RA. We adjusted our estimates for a wide range of confounding factors using a multivariable regression model. Missing information on BMI was considered as a separate category and included in the analysis. Recency of antibiotic use was assessed as the time between antibiotic therapy end date and the occurrence of RA. Using similar methods, we assessed the impact of antibiotic type (bacteriostatic/ bactericidal; Additional file 1: Table S1) on RA onset.

To explore whether the underlying infection, rather than the antibiotic prescription, is associated with increased risk of RA (confounding by indication), the strength of association between previous infections and RA was assessed and stratified by infection types. Frequency-dependent associations (number of previous infections) with RA were also explored. For this purpose, similar infections recorded less than 30 days apart were considered as a single episode. As upper respiratory tract (URT) infections may or may not warrant antibiotic therapy, we stratified our analysis by treatment modality (antibiotic-treated URT or untreated 
URT). Treated URT was defined as those prescribed any antibiotics within 30 days of their initial infection code. For each patient, we counted the number of antibiotic-treated and untreated URT infections before the index date and assessed the association with RA. For comparison, we assessed the association between non-bacterial antimicrobial agents (anti-fungal and anti-viral) and the risk of RA.

It may be possible that certain early RA symptoms may have triggered antibiotic therapy (protopathic bias). To account for this bias, we repeated our primary analysis after redefining our index date based on patients' first referrals to rheumatology clinics within 5 years before their recorded RA diagnosis (similar date was imposed on matched controls). All statistical analysis was conducted using Stata version 14.

\section{Approvals}

This study was approved by the CPRD's Independent Scientific Advisory Committee (ISAC) reference number: 18_245.

\section{Results}

\section{Participant characteristics}

Twenty-two thousand six hundred seventy-seven cases of incident RA cases were individually matched to 90,013 controls. Baseline characteristics of the study population are summarised in Table 1. Compared to controls, a larger proportion of RA cases were obese (BMI > 30; $27 \%$ vs. $32 \%$ ), were more likely to smoke ( $18 \%$ vs. $21 \%$ ), and had a higher number of comorbidities (no comorbidities $63 \%$ vs. $57 \%$ ).

\section{Antibiotics and RA}

RA cases received more antibiotic prescriptions than controls, but over $80 \%$ in each group had received a prescription in the 10 years before the index date (Table 2). Penicillins were the most commonly prescribed antibiotics (72\% of participants received at least one prescription before index data), followed by macrolides (33\%), trimethoprim (31\%), cephalosporins (25\%), and tetracyclines and quinolones (both 13\%). After adjusting for potential confounding factors, the odds of having been exposed to antibiotics were significantly higher in RA cases than in controls (OR 1.60; 95\% CI 1.51-1.68). Redefining our index date based on the first referral to the rheumatology did not change our overall finding (adjusted $\mathrm{OR}=1.68$; 95\% CI 1.59-1.76). A frequency-dependent relationship was observed between the number of prescriptions and the odds of RA. The magnitude of association with RA increased with an increasing number of unique antibiotic prescriptions. The timing of antibiotic exposure was important, with higher odds of RA in those last exposed to antibiotics in 1 to 2 years before the index date (OR 1.80; 95\% CI 1.70-1.90) than in those last exposed between 5 and 10 years before the index date $(\mathrm{OR}=1.57 ; 95 \% \mathrm{CI}$ 1.49-1.66).

All classes of antibiotics were associated with higher odds of RA, with clindamycin having the highest odds ratio (Fig. 1). Based on the mode-of-action, the odds of RA were $45 \%$ higher in those prescribed antibiotics inducing a bactericidal response (i.e. lyse bacteria) (OR = 1.45 ; $95 \%$ CI 1.39-1.51) compared to those not prescribed antibiotics. The increase in odds was slightly lower with antibiotics inhibiting bacteria (i.e. bacteriostatic) when similar comparisons were made $(\mathrm{OR}=1.31$; 95\% CI 1.27-1.36).

\section{RA and infection}

Cases were more likely to have a record of infection before their RA diagnosis (Table 3). This increased odds of RA among those with a previous history of infection ranged between $7 \%$ (urinary tract infection; $\mathrm{OR}=1.07$; 95\% CI 1.02-1.12) and 37\% (for LRT infections; OR = 1.37; 95\% CI 1.32-1.41). A frequency-dependent association also existed between the number of previous infections and the odds of RA. More than $87 \%$ of lower respiratory and urinary tract infections were treated with antibiotics within 30 days. This proportion was lower for URT (74\%) and GI infections (21\%). Those with antibiotic-treated URT infections were more likely to be RA cases. However, the association was not observed for the number of untreated URT infections.

\section{Additional analysis}

A total of 1580 (7\%) and 3166 (14\%) of RA cases received at least one antiviral and antifungal prescription, respectively, before the index date. After adjusting for confounding factors, including antibiotic use, both antifungal $(\mathrm{OR}=1.19 ; 95 \% \mathrm{CI} 1.14-1.24)$ and antiviral drugs $(\mathrm{OR}=1.27$; 95\% CI 1.20-1.35) were associated with increased risk of $\mathrm{RA}$.

\section{Discussion}

Using routinely collected primary care data, we have assessed the association between antibiotic use and RA onset. Those exposed to one or more antibiotic prescriptions were $60 \%$ more likely to develop RA than their unexposed counterparts. A frequency-dependent association was observed between previous antibiotic prescriptions and RA, and the odds of RA were higher in those with more recent antibiotic exposure. The odds of RA varied by class and mode-of-action of antibiotics. Infection type was also important; those with antibiotic-treated URT infections were more likely to be RA cases. However, the same association was not observed for the number of untreated URT infections. Finally, other anti-microbial agents (i.e. antifungals and antivirals) were associated with increased RA risk. 
Table 1 Basic characteristics of cases and controls

\begin{tabular}{|c|c|c|c|c|}
\hline \multirow[t]{2}{*}{ Variable } & \multicolumn{2}{|c|}{ Controls $(n=90,013)$} & \multicolumn{2}{|c|}{ RA cases $(n=22,677)$} \\
\hline & $N$ & $\%$ & $N$ & $\%$ \\
\hline \multicolumn{5}{|l|}{ Year of RA diagnosis } \\
\hline 1995-1999 & 8086 & 9 & 2033 & 9 \\
\hline 2000-2004 & 15,253 & 16.9 & 3849 & 17 \\
\hline 2005-2009 & 24,713 & 27.5 & 6232 & 27.5 \\
\hline 2010-2014 & 31,699 & 35.2 & 7980 & 35.2 \\
\hline 2015-2017 & 10,262 & 11.4 & 2583 & 11.4 \\
\hline \multicolumn{5}{|l|}{ Age at index (years) } \\
\hline$<40$ & 19,064 & 21.2 & 4857 & 21.4 \\
\hline $40-59$ & 20,416 & 22.7 & 5095 & 22.5 \\
\hline $60-69$ & 22,257 & 24.7 & 5588 & 24.6 \\
\hline $70-79$ & 19,238 & 21.4 & 4843 & 21.4 \\
\hline $80-89$ & 8366 & 9.3 & 2093 & 9.2 \\
\hline$\geq 90$ & 672 & 0.7 & 201 & 0.9 \\
\hline Mean age (SD) & 61.4 & $(14.1)$ & 61.4 & $(14.3)$ \\
\hline Gender, female & 60,620 & 67.3 & 15,276 & 67.4 \\
\hline Median follow-up before RA diagnosis (IQR) & 10 & $(7-14)$ & 10 & $(7-14)$ \\
\hline \multicolumn{5}{|l|}{ Body mass index } \\
\hline Underweight & 22,337 & 24.8 & 5102 & 22.5 \\
\hline Normal & 2402 & 2.7 & 694 & 3.1 \\
\hline Overweight & 28,948 & 32.2 & 7456 & 32.9 \\
\hline Obese & 24,610 & 27.3 & 7308 & 32.2 \\
\hline Missing & 11,716 & 13 & 2117 & 9.3 \\
\hline \multicolumn{5}{|l|}{ Smoking status } \\
\hline Never/ex-smokers & 74,043 & 82.3 & 17,840 & 78.7 \\
\hline Current & 15,970 & 17.7 & 4837 & 21.3 \\
\hline \multicolumn{5}{|l|}{ Alcohol } \\
\hline Never/ex-drinker & 29,159 & 32.4 & 7341 & 32.4 \\
\hline $1-10$ units/weeks & 46,692 & 51.9 & 11,976 & 52.8 \\
\hline$>10$ units/week & 14,162 & 15.7 & 3360 & 14.8 \\
\hline \multicolumn{5}{|l|}{ Charlson comorbidity index } \\
\hline 0 & 56,683 & 63 & 12,813 & 56.5 \\
\hline $1-2$ & 24,356 & 27.1 & 7219 & 31.8 \\
\hline $3-5$ & 7937 & 8.8 & 2355 & 10.4 \\
\hline$>5$ & 1037 & 1.2 & 290 & 1.3 \\
\hline
\end{tabular}

Respiratory infections showed the strongest association with RA. Chlamydia pneumoniae, Streptococcus pyogenes, Streptococcus pneumoniae, and Klebsiella pneumoniae are often associated with respiratory tract infections, and previous studies have linked specific pathogens (e.g. C. pneumoniae) with increased levels of circulating autoimmune antibodies that may play a role in RA pathology [22]. However, as numerous pathogens are associated with URT infections, other factors may also play a strong role in disease association. Indeed, our analysis indicates the strongest association is only present in antibiotic-treated cases of URTs (74\% patients treated within 30 days of diagnosis), not untreated URT infections, suggesting that associated antibiotic usage is likely to be the main associative factor for the increased incidence of RA in this cohort. A previous case-control study in JIA also suggested that antibiotic-treated URT infections were more strongly associated with JIA than untreated URT infections [8]. It should be noted that antibiotic-treated respiratory infections may be expected 
Table 2 Association between antibiotic prescriptions and RA

\begin{tabular}{|c|c|c|c|c|c|c|}
\hline \multirow[t]{2}{*}{ Variables } & \multicolumn{2}{|c|}{ Controls } & \multicolumn{2}{|l|}{ Cases } & \multirow{2}{*}{$\begin{array}{l}\text { Crude OR (95\% } \\
\text { Cl) }\end{array}$} & \multirow{2}{*}{$\begin{array}{l}\text { Adjusted } \mathrm{OR}^{\mathrm{a}} \\
(95 \% \mathrm{Cl})\end{array}$} \\
\hline & $\bar{N}$ & $\%$ & $\bar{N}$ & $\%$ & & \\
\hline \multicolumn{7}{|l|}{ Any antibiotic use } \\
\hline No prescriptions & 16,242 & 18.0 & 2369 & 10.4 & Reference & Reference \\
\hline$\geq 1$ prescription & 73,771 & 82.0 & 20,308 & 89.6 & $2.02(1.92-2.12)$ & $1.60(1.51-1.68)$ \\
\hline \multicolumn{7}{|c|}{ No. of prescriptions (quartile) (median (IQR)) } \\
\hline $1(1(1,2))$ & 20,321 & 22.6 & 4128 & 18.2 & $1.49(1.41-1.57)$ & $1.40(1.32-1.48)$ \\
\hline $2(4(3,5))$ & 18,923 & 21.0 & 4592 & 20.2 & $1.85(1.75-1.96)$ & $1.66(1.57-1.77)$ \\
\hline $3(8(7,9))$ & 17,524 & 19.5 & 5231 & 23.1 & $2.36(2.23-2.50)$ & $2.04(1.92-2.17)$ \\
\hline $4(19(14,29))$ & 17,003 & 18.9 & 6357 & 28.0 & $3.10(2.93-3.28)$ & $2.56(2.39-2.74)$ \\
\hline \multicolumn{7}{|c|}{ Recency of therapy (therapy termination) ${ }^{b}$} \\
\hline $1-2$ years before diagnosis & 30,041 & 35.2 & 9557 & 43.9 & $2.34(2.23-2.46)$ & $1.80(1.70-1.90)$ \\
\hline $2-5$ years before diagnosis & 26,898 & 31.5 & 7016 & 32.2 & $1.91(1.81-2.01)$ & $1.57(1.49-1.66)$ \\
\hline $5-10$ years before diagnosis & 12,183 & 14.3 & 2846 & 13.1 & $1.70(1.59-1.80)$ & $1.48(1.39-1.58)$ \\
\hline \multicolumn{7}{|l|}{ Antibiotic type } \\
\hline \multicolumn{7}{|l|}{ Bacteriostatic } \\
\hline Unexposed $^{c}$ & 43,580 & 48.4 & 8778 & 38.7 & Reference & Reference \\
\hline Exposed & 46,433 & 51.6 & 13,899 & 61.3 & $1.55(1.50-1.60)$ & $1.31(1.27-1.36)$ \\
\hline \multicolumn{7}{|l|}{ Bactericidal } \\
\hline Unexposed $^{d}$ & 21,892 & 24.3 & 3595 & 15.9 & Reference & Reference \\
\hline Exposed & 68,121 & 75.7 & 19,082 & 84.2 & $1.80(1.72-1.87)$ & $1.45(1.39-1.51)$ \\
\hline
\end{tabular}

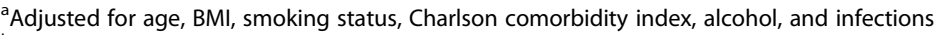

${ }^{b}$ Most recent course of therapy

Including those people exposed only to bactericidal antibiotics

${ }^{d}$ Including those people exposed only to bacteriostatic antibiotics

to be more severe, thus potentially confounding analysis; nonetheless, the link between the severity of an infection and antibiotic prescription practices is not currently clear. Whilst there is an expectation that this should be the case, numerous previous studies have highlighted that clinician-dependent decisions and preference are the likely driver for an antibiotic prescription for infections that notably are rarely confirmed by microbiological testing (with many cases potentially being of viral origin) [23, 24]. Further analysis of antibiotic usage indicates that all classes increase the risk of RA development, which suggests that provision of antibiotics rather

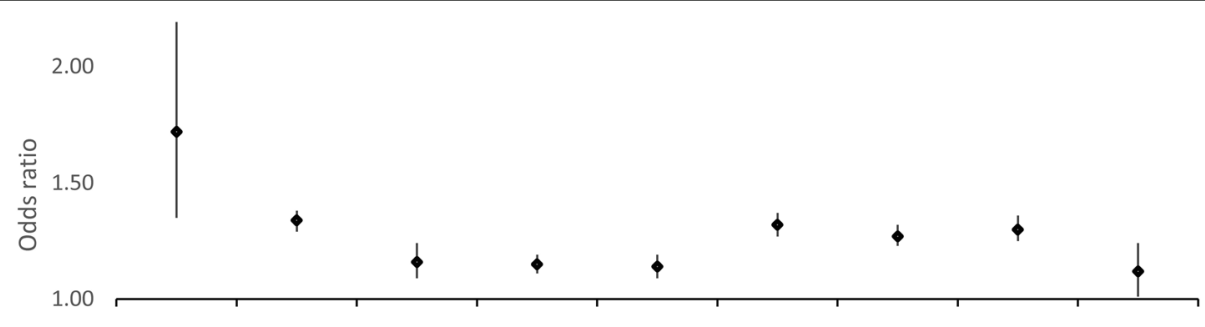

0.50

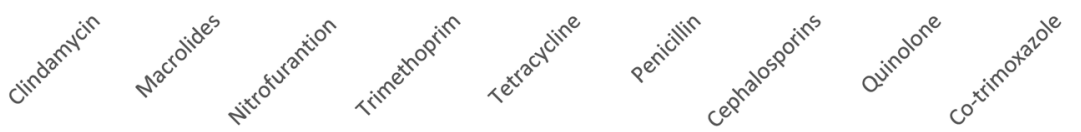

Antibiotic type

Fig. 1 Association between RA and antibiotic class. Comparison of individual antibiotic classes with no antibiotics. Estimates are adjusted for Charlson index, age category, BMI category, smoking, alcohol consumption, and infection types 
Table 3 Association between the history of infections and RA

\begin{tabular}{|c|c|c|c|c|c|c|}
\hline \multirow[t]{2}{*}{ Variables } & \multicolumn{2}{|c|}{ Controls } & \multicolumn{2}{|l|}{ Cases } & \multirow[t]{2}{*}{ OR $(95 \% \mathrm{Cl})$} & \multirow{2}{*}{$\begin{array}{l}\text { Adjusted } \mathrm{OR}^{*} \\
(95 \% \mathrm{Cl})\end{array}$} \\
\hline & $N$ & $\%$ & $N$ & $\%$ & & \\
\hline \multicolumn{7}{|c|}{ Infection ever before diagnosis } \\
\hline Lower respiratory tract & 31,400 & 34.9 & 10,050 & 44.3 & $1.56(1.51-1.61)$ & $1.37(1.32-1.41)$ \\
\hline Upper respiratory tract & 41,786 & 46.4 & 12,258 & 54.1 & $1.42(1.38-1.47)$ & $1.26(1.22-1.31)$ \\
\hline Gastrointestinal & 8352 & 9.3 & 2699 & 11.9 & $1.33(1.27-1.40)$ & $1.19(1.13-1.25)$ \\
\hline Urinary tract & 11,037 & 12.3 & 3179 & 14 & $1.19(1.13-1.24)$ & $1.07(1.02-1.12)$ \\
\hline \multicolumn{7}{|l|}{ Infection episodes } \\
\hline 0 & 34,899 & 38.8 & 6835 & 30.1 & Reference & Reference \\
\hline 1 & 18,503 & 20.6 & 4366 & 19.3 & $1.26(1.21-1.32)$ & $1.22(1.72-1.28)$ \\
\hline 2 & 11,041 & 12.3 & 2990 & 13.2 & $1.48(1.41-1.56)$ & $1.42(1.35-1.49)$ \\
\hline 3 & 7091 & 7.9 & 2066 & 9.1 & $1.63(1.54-1.73)$ & $1.54(1.46-1.64)$ \\
\hline$\geq 4$ & 18,479 & 20.5 & 6420 & 28.3 & $2.02(1.93-2.11)$ & $1.86(1.78-1.94)$ \\
\hline \multicolumn{7}{|l|}{ No. of treated URT } \\
\hline 0 & 8466 & 20.3 & 2078 & 17.0 & Reference & Reference \\
\hline 1 & 16,536 & 39.6 & 4510 & 36.8 & $1.11(1.05-1.18)$ & $1.10(1.04-1.17)$ \\
\hline 2 & 7052 & 16.9 & 2236 & 18.2 & $1.29(1.21-1.38)$ & $1.27(1.19-1.50)$ \\
\hline 3 & 3554 & 8.5 & 1233 & 10.1 & $1.41(1.30-1.53)$ & $1.39(1.28-1.51)$ \\
\hline$\geq 4$ & 6178 & 14.8 & 2201 & 18.0 & $1.45(1.36-1.55)$ & $1.42(1.32-1.52)$ \\
\hline \multicolumn{7}{|c|}{ No. of untreated URT } \\
\hline 0 & 21,981 & 52.6 & 6510 & 53.1 & Reference & Reference \\
\hline 1 & 13,893 & 33.2 & 3908 & 31.9 & $0.95(0.91-0.99)$ & $0.95(0.91-1.00)$ \\
\hline 2 & 3714 & 8.9 & 1132 & 9.2 & $1.03(0.96-1.11)$ & $1.02(0.95-1.07)$ \\
\hline 3 & 1274 & 3 & 385 & 3.1 & $1.02(0.91-1.15)$ & $1.00(0.89-1.13)$ \\
\hline$\geq 4$ & 924 & 2.2 & 323 & 2.6 & $1.18(1.04-1.34)$ & $1.14(1.00-1.29)$ \\
\hline
\end{tabular}

*Adjusted for age, BMI, smoking status, Charlson comorbidity index, alcohol, and presence/absence of LRT, URT, GII, and UTI

than type/class is associated with RA. The odds ratio for clindamycin was the largest, which was also observed by Arvonen et al. [10] in JIA patients. However, the confidence interval is very wide, which reflects the small numbers of people prescribed with this antibiotic $(n=307,0.27 \%$ of the sample); thus, this medication is not expected to have a strong influence on the overall association of antibiotic prescription with RA diagnosis. Breaking down antibiotics into their mode-of-action revealed that there was a stronger association of bactericidals than bacteriostatics with RA (OR 1.45 vs. 1.31), which may correlate with increased gut microbiota disturbances and link to our observation that antibiotic exposure up to 10 years prior to diagnosis was associated with increased risk of RA [5]. Lysis of bacteria by bactericidal antibiotics may also be associated with further microbial compounds circulating systemically and inducing autoimmune antibodies that are associated with RA pathology. However, it is currently unclear if the major driver is infection and/or antibiotic usage; therefore, these observations require extensive additional studies (both case-controlled and mechanistic) to determine direct causation.
The rise in inflammatory, allergic, and autoimmune conditions over the past 20-30 years has been linked to improved diagnostics and a more 'Western' lifestyle, which includes the widespread use of antibiotics [9]. Importantly, these antibiotics, whilst crucial in treating serious bacterial pathogens, also affect beneficial microbiota members, leading to a reduction in microbial diversity. In some cases, these disturbances appear to be longer term, whilst in others, the microbiota appears to revert to baseline $[25,26]$. However, even short-term changes may interrupt the key immune pathways, which may manifest as symptoms in later-life conditions. Indeed, there is now strong evidence linking antibiotic-induced microbiota disturbances to numerous pathologies. Indeed, IBD is defined as 'a chronic intestinal inflammation that results from host-microbial interactions in a genetically susceptible individual', and previous studies have indicated a sevenfold higher risk of IBD development in response to antibiotic use [27]. Furthermore, many mechanistic preclinical studies have provided strong evidence that an antibiotic-disturbed gut microbiota plays a direct role in disease 
pathology [28]. Similarly, alongside the genetic susceptibility component to RA, it is apparent that additional environmental factors, including microbes, may play a role in disease onset. Horton et al. performed a case-controlled study of antibiotic usage and JIA that indicated that any antibiotic exposure was associated with increased risk of developing JIA [8]. This association was also the frequency of antibiotic use-dependent, supporting our findings that inflammatory arthritis may be associated with antibioticrelated gut microbiota disturbances. Our study is the first to investigate the association between antibiotic usage and RA and suggests that antibiotics may be a major risk factor for RA development.

Small scale studies profiling the microbiota of RA patients have indicated reduced bacterial taxon diversity. A study from the mid-1990s focussing on microbial-derived compounds, i.e. cellular fatty acids, indicated a difference between RA patients and controls, suggesting a microbiota component to RA pathology [29]. Other studies using 16S rRNA microbiota analysis have indicated changes in different bacterial genus ranging from reductions in Bacteroides, Prevotella, and Porphyromonas to another study indicating that a subpopulation of early RA patients had increased prevalence of Prevotella copri; however, it should be noted that these observed microbiome changes may also be a result of RA disease state [30, 31]. Using more sensitive microbiota profiling approaches, i.e. shotgun metagenomics (involves sequencing the entire gene content of microbiota samples), Zhang et al. determined that both oral and gut microbial communities are altered in RA patients, with Bifidobacterium bifidum and Haemophilus spp. reduced and negatively correlated with the levels of serum autoantibodies, whereas Lactobacillus salivarius was over-represented, particularly in patients with very active RA [17]. A lung microbiota study, using metataxonomic $16 \mathrm{~S}$ rRNA profiling, indicated that bronchoalveolar lavage fluid (BAL) from RA patients had reduced species diversity, which correlated with RA-associated lung pathology, and suggests that lung microbiota changes may also be associated with clinical outcomes [32]. However, all these studies were carried out in small numbers of patients $(<80)$, and none has investigated the association with antibiotic use, thus highlighting the need for further studies in this area.

Alongside bacteria, the microbiota also includes fungi and viruses, and more recent studies have indicated that these microorganisms play a key role in microbial ecosystem structuring and modulating host immune responses [33, 34]. Thus, our findings that increased risk of RA was also observed for patients prescribed other anti-microbial agents (i.e. antifungals, antivirals), albeit a lower risk than antibiotics, suggests that the risk may be in part driven by disturbances in these microbial groups, which warrants further exploration. Additional analysis to assess the potential confounding effect of antifungals and antivirals (data not shown) suggested that prescriptions of the classes of medication were independent of each other.

Our study has a number of limitations. First, there may be misclassification of RA cases, as we were reliant on general practitioners accurately record RA diagnosis. However, previous studies have shown high accuracy of RA recording in primary care; therefore, the risk of misclassification will be minimal $[20,21]$. On that note, we considered incident RA cases based on the earliest recording of RA in the patients' primary care record. This may sometimes not reflect the true date of RA onset as it should be diagnosed by a specialist rheumatologist. Any delay in the recording of RA in primary care data may lead to protopathic bias. However, our finding remained unchanged when we conducted a sensitivity analysis after redefining our index date based on the first referral to a rheumatologist. Second, factors such as parity and breastfeeding are associated with increased risk of RA, which may have confounded our observed association. However, the impact of this confounding will be limited, as the onset of RA is relatively rare in the younger population. Third, we may have underestimated the proportion of individuals exposed to antibiotics, as we did not have information on antibiotics prescribed in secondary care. Given that we found that patients with RA have more comorbidities compared to controls, it is reasonable to assume that RA cases may have had more hospital admissions and subsequently received more secondary care prescriptions. Thus, we may have underestimated the association between antibiotic prescriptions and RA. In addition, there may be confounding by the underlying infection. Whilst increased risk of RA was not observed for those with URT infections not treated with antibiotics, this finding should be interpreted with caution as antibiotics may be a marker of infection severity (albeit with the caveats highlighted above). Finally, we did not determine relative doses of antibiotics taken; therefore, we used frequency of prescriptions to correlate with potential dose and other commonly prescribed drugs, e.g. proton pump inhibitors may be correlated with antibiotic use and in turn confound the observed influence on RA risk (and the microbiota), with additional studies required to explore these complex issues [35]. This finding cannot be generalised to other infections as we have shown that the risk of RA varies by the infection site.

Although we have identified a strong association between antibiotic usage and the onset of RA, there remain many unanswered questions, particularly whether this is related to infections themselves, alterations in the microbiota, or a combination of the two. Further clinical information on the causative pathogens, and their association 
with the risk of RA, may shed light on this, and further larger microbiota profiling studies, using cutting edge sequencing approaches, may provide a further resolution on the microbes present or absent and links to specific microbial components. However, these studies should also be underpinned by mechanistic investigations that help researchers define what processes are involved, providing an important platform for the development of new prevention or intervention strategies. Indeed, with the identification of microbiota disturbances in several human disease pathologies, modulation of the microbiota has become a target for intervention and treatment. This may also prove to be useful in the context of RA, although $80 \%$ of people had a recorded prescription for an antibiotic, the odds of having received an antibiotic were $60 \%$ higher in those with RA. To date, there have been a limited number of randomised clinical trials using probiotics (live organisms which, when administered in adequate amounts, confer a health benefit on the host), to treat RA, and outcomes have been inconsistent [36]. However, immune readouts indicate a reduction in inflammatory cytokines (TNF- $\alpha$ and IL-8) and a concurrent increase in IL-10 (an anti-inflammatory cytokine) [37]. We feel this is an important study to highlight other aetiological factors, such as antibiotics, that may contribute to RA risk. Studies of this nature, profiling a huge longitudinal population base, are critical for next stage detailed clinical studies and development of innovative therapies for this debilitating disease, including probing underlying mechanisms and potential associations between microbiota disturbances, infection, and RA onset.

\section{Additional file}

Additional file 1: Table S1. Categorisation of antibiotics. (DOCX $13 \mathrm{~kb}$ )

\author{
Abbreviations \\ ACPA: Antibodies to citrullinated peptide antigens; BAL: Bronchoalveolar \\ lavage fluid; BMI: Body mass index; BNF: British National Formulary; \\ Cl: Confidence interval; CPRD: Clinical Practice Research Datalink; \\ GI: Gastrointestinal; IQR: Interquartile range; ISAC: Independent Scientific \\ Advisory Committee; JA: Juvenile inflammatory arthritis; LRT: Lower \\ respiratory tract; OR: Odds ratio; RA: Rheumatoid arthritis; SD: Standard \\ deviation; TNF: Tumour necrosis factor; URT: Upper respiratory tract; UTS: Up \\ to standard
}

\section{Acknowledgements}

This study is based in part on data from the Clinical Practice Research Datalink obtained under licence from the UK Medicines and Healthcare products Regulatory Agency. The data is provided by patients and collected by the NHS as part of their care and support. The interpretation and conclusions contained in this study are those of the authors alone.

\section{Authors' contributions}

This study was initially conceived of and preliminarily designed by CM and LJH. The analysis was undertaken by AAS and SM. The manuscript was authored and approved by all named authors.

\section{Funding}

CDM is funded by a National Institute for Health Research (NIHR) Research Professorship (NIHR-RP- 2014-04- 026) which also funded AAS. CDM is also funded by the NIHR Collaborations for Leadership in Applied Health Research and Care West Midlands and the NIHR School for Primary Care Research. TH is funded by an NIHR Clinical Lectureship.

This work was also supported by a Wellcome Trust Investigator Award (100974/C/13/Z) and the Biotechnology and Biological Sciences Research Council (BBSRC) Institute Strategic Programme Gut Microbes and Health BB/ R012490/1 and its constituent project(s) BBS/E/F/000PR10353 and BBS/E/F/ 000PR10356 to LJH.

The study sponsors had no role in the study design, collection, analysis and interpretation of data, writing of the report, or decision to submit the paper for publication. The views and opinions expressed herein are those of the authors and do not necessarily reflect those of the NIHR (UK). This paper presents independent research which is part-funded by the CLAHRC West Midlands. The views expressed are those of the authors and not necessarily those of the NHS, the NIHR, or the Department of Health and Social Care.

\section{Availability of data and materials}

CPRD data were analysed under licence and are not available for public sharing. Access to CPRD is available to all qualified researchers via procedures detailed on the CPRD website (https://www.cprd.com/home/). Applications for data access are submitted to isac@cprd.com.

Ethics approval and consent to participate

Not applicable

\section{Consent for publication}

Not applicable

\section{Competing interests}

The authors declare that they have no competing interests.

\section{Author details}

${ }^{1}$ Arthritis Research UK Primary Care Centre, Institute for Primary care and Health Sciences, Keele University, Keele ST5 5BG, UK. ${ }^{2}$ Haywood Academic Rheumatology Centre, Midlands Partnership Foundation Trust, Staffordshire ST6 7AG, UK. ${ }^{3}$ Gut Microbes \& Health Programme, Quadram Institute Bioscience, Norwich Research Park, Norwich NR4 7UQ, UK.

Received: 21 May 2019 Accepted: 17 July 2019

Published online: 07 August 2019

\section{References}

1. Dolk FCK, Pouwels KB, Smith DRM, Robotham JV, Smieszek T. Antibiotics in primary care in England: which antibiotics are prescribed and for which conditions? J Antimicrob Chemother. 2018;73(suppl_2):ii2-ii10.

2. Cianci R, Pagliari D, Piccirillo CA, Fritz JH, Gambassi G. The microbiota and immune system crosstalk in health and disease. Mediat Inflamm. 2018;2018: 2912539.

3. O'Neill ISZ, Hall LJ. Exploring the role of the microbiota member Bifidobacterium in modulating immune-linked diseases. Emerg Top Life Sci. 2017;1:333-49.

4. Rowland I, Gibson G, Heinken A, Scott K, Swann J, Thiele I, Tuohy K. Gut microbiota functions: metabolism of nutrients and other food components. Eur J Nutr. 2018:57(1):1-24.

5. laniro G, Tilg H, Gasbarrini A. Antibiotics as deep modulators of gut microbiota: between good and evil. Gut. 2016;65(11):1906-15.

6. Blaser MJ. Antibiotic use and its consequences for the normal microbiome. Science. 2016;352(6285):544-5.

7. Verwoerd A, Ter Haar NM, de Roock S, Vastert SJ, Bogaert D. The human microbiome and juvenile idiopathic arthritis. Pediatr Rheumatol Online J. 2016;14(1):55.

8. Horton DB, Scott Fl, Haynes K, Putt ME, Rose CD, Lewis JD, Strom BL. Antibiotic exposure and juvenile idiopathic arthritis: a case-control study. Pediatrics. 2015;136(2):e333-43.

9. Shamriz O, Mizrahi H, Werbner M, Shoenfeld Y, Avni O, Koren O. Microbiota at the crossroads of autoimmunity. Autoimmun Rev. 2016;15(9):859-69.

10. Arvonen M, Virta LJ, Pokka T, Kroger L, Vahasalo P. Repeated exposure to antibiotics in infancy: a predisposing factor for juvenile idiopathic arthritis or 
a sign of this group's greater susceptibility to infections? J Rheumatol. 2015; 42(3):521-6.

11. Smolen JS, Aletaha D, Barton A, Burmester GR, Emery P, Firestein GS, Kavanaugh A, Mclnnes IB, Solomon DH, Strand V, et al. Rheumatoid arthritis. Nat Rev Dis Primers. 2018;4:18001.

12. Konig MF, Abusleme L, Reinholdt J, Palmer RJ, Teles RP, Sampson K, Rosen A, Nigrovic PA, Sokolove J, Giles JT, et al. Aggregatibacter actinomycetemcomitans-induced hypercitrullination links periodontal infection to autoimmunity in rheumatoid arthritis. Sci Transl Med. 2016; 8(369):369ra176.

13. Ayravainen L, Leirisalo-Repo M, Kuuliala A, Ahola K, Koivuniemi R, Meurman $\mathrm{JH}$, Heikkinen AM. Periodontitis in early and chronic rheumatoid arthritis: a prospective follow-up study in Finnish population. BMJ Open. 2017;7(1): e011916.

14. Chapple ILC, Van der Weijden F, Doerfer C, Herrera D, Shapira L, Polak D, Madianos P, Louropoulou A, Machtei E, Donos N, et al. Primary prevention of periodontitis: managing gingivitis. J Clin Periodontol. 2015;42:S71-6,

15. Scher JU, Littman DR, Abramson SB. Microbiome in inflammatory arthritis and human rheumatic diseases. Arthritis Rheumatol. 2016;68(1):35-45.

16. Rogier R, Evans-Marin H, Manasson J, van der Kraan PM, Walgreen B, Helsen $M M$, van den Bersselaar LA, van de Loo FA, van Lent PL, Abramson SB, et al. Alteration of the intestinal microbiome characterizes preclinical inflammatory arthritis in mice and its modulation attenuates established arthritis. Sci Rep. 2017;7(1):15613.

17. Zhang X, Zhang D, Jia H, Feng Q, Wang D, Liang D, Wu X, Li J, Tang L, Li Y, et al. The oral and gut microbiomes are perturbed in rheumatoid arthritis and partly normalized after treatment. Nat Med. 2015;21(8):895-905.

18. Maeda Y, Kurakawa T, Umemoto E, Motooka D, Ito Y, Gotoh K, Hirota K, Matsushita M, Furuta Y, Narazaki M, et al. Dysbiosis contributes to arthritis development via activation of autoreactive T cells in the intestine. Arthritis Rheumatol. 2016;68(11):2646-61.

19. Herrett $\mathrm{E}$, Gallagher AM, Bhaskaran $\mathrm{K}$, Forbes $H$, Mathur R, van Staa T, Smeeth L. Data resource profile: Clinical Practice Research Datalink (CPRD). Int J Epidemiol. 2015;44(3):827-36.

20. Muller S, Hider SL, Raza K, Stack RJ, Hayward RA, Mallen CD. An algorithm to identify rheumatoid arthritis in primary care: a Clinical Practice Research Datalink study. BMJ Open. 2015;5(12):e009309.

21. Thomas SL, Edwards CJ, Smeeth L, Cooper C, Hall AJ. How accurate are diagnoses for rheumatoid arthritis and juvenile idiopathic arthritis in the general practice research database? Arthritis Rheum. 2008;59(9):1314-21.

22. Fujita M, Hatachi S, Yagita M. Acute Chlamydia pneumoniae infection in the pathogenesis of autoimmune diseases. Lupus. 2009;18(2):164-8.

23. Jones BE, Sauer B, Jones MM, Campo J, Damal K, He T, Ying J, Greene T, Goetz MB, Neuhauser MM, et al. Variation in outpatient antibiotic prescribing for acute respiratory infections in the veteran population a cross-sectional study. Ann Intern Med. 2015;163(2):73.

24. Fleming-Dutra KE, Bartoces M, Roberts RM, Hicks LA. Characteristics of primary care physicians associated with high outpatient antibiotic prescribing volume. Open Forum Infect Dis. 2018;5(1):ofx279.

25. Yassour M, Vatanen T, Siljander H, Hamalainen AM, Harkonen T, Ryhanen SJ, Franzosa EA, Vlamakis H, Huttenhower C, Gevers D, et al. Natural history of the infant gut microbiome and impact of antibiotic treatment on bacterial strain diversity and stability. Sci Transl Med. 2016;8(343):343ra381.

26. Hildebrand F, Moitinho-Silva L, Blasche S, Jahn MT, Gossmann TI, HeuertaCepas J, Hercog R, Luetge M, Bahram M, Pryszlak A, et al. Antibioticsinduced monodominance of a novel gut bacterial order. Gut. 2019. https:// doi.org/10.1136/gutjil-2018-317715.

27. Kronman MP, Zaoutis TE, Haynes K, Feng R, Coffin SE. Antibiotic exposure and IBD development among children: a population-based cohort study. Pediatrics. 2012;130(4):e794-803.

28. Schulfer AF, Battaglia T, Alvarez Y, Bijnens L, Ruiz VE, Ho M, Robinson S, Ward $T$, Cox $L M$, Rogers $A B$, et al. Intergenerational transfer of antibioticperturbed microbiota enhances colitis in susceptible mice. Nat Microbiol. 2018;3(2):234-42.

29. Eerola E, Mottonen T, Hannonen P, Luukkainen R, Kantola I, Vuori K, Tuominen J, Toivanen P. Intestinal flora in early rheumatoid arthritis. Br J Rheumatol. 1994;33(11):1030-8

30. Scher JU, Sczesnak A, Longman RS, Segata N, Ubeda C, Bielski C, Rostron T, Cerundolo V, Pamer EG, Abramson SB, et al. Expansion of intestinal Prevotella copri correlates with enhanced susceptibility to arthritis. Elife. 2013;2:e01202
31. Vaahtovuo J, Munukka E, Korkeamaki M, Luukkainen R, Toivanen P. Feca microbiota in early rheumatoid arthritis. J Rheumatol. 2008;35(8):1500-5.

32. Scher JU, Joshua V, Artacho A, Abdollahi-Roodsaz S, Ockinger J, Kullberg S, Skold M, Eklund A, Grunewald J, Clemente JC, et al. The lung microbiota in early rheumatoid arthritis and autoimmunity. Microbiome. 2016;4(1):60.

33. Paterson MJ, Oh S, Underhill DM. Host-microbe interactions: commensal fungi in the gut. Curr Opin Microbiol. 2017;40:131-7.

34. Freer G, Maggi F, Pifferi M, Di Cicco ME, Peroni DG, Pistello M. The virome and its major component, Anellovirus, a convoluted system molding human immune defenses and possibly affecting the development of asthma and respiratory diseases in childhood. Front Microbiol. 2018;9:686.

35. Jackson MA, Goodrich JK, Maxan ME, Freedberg DE, Abrams JA, Poole AC, Sutter JL, Welter D, Ley RE, Bell JT, et al. Proton pump inhibitors alter the composition of the gut microbiota. Gut. 2016;65(5):749-56.

36. Hill C, Guarner F, Reid G, Gibson GR, Merenstein DJ, Pot B, Morelli L, Canani $\mathrm{RB}$, Flint HJ, Salminen S, et al. Expert consensus document. The International Scientific Association for Probiotics and Prebiotics consensus statement on the scope and appropriate use of the term probiotic. Nat Rev Gastroenterol Hepatol. 2014;11(8):506-14.

37. Pan HD, Li RZ, Li T, Wang J, Liu L. Whether probiotic supplementation benefits rheumatoid arthritis patients: a systematic review and metaanalysis. Engineering Prc. 2017;3(1):115-21.

\section{Publisher's Note}

Springer Nature remains neutral with regard to jurisdictional claims in published maps and institutional affiliations.
Ready to submit your research? Choose BMC and benefit from:

- fast, convenient online submission

- thorough peer review by experienced researchers in your field

- rapid publication on acceptance

- support for research data, including large and complex data types

- gold Open Access which fosters wider collaboration and increased citations

- maximum visibility for your research: over $100 \mathrm{M}$ website views per year

At BMC, research is always in progress.

Learn more biomedcentral.com/submissions 